SUBJECT AREAS: OPTICS AND PHOTONICS OPTICAL MATERIALS AND STRUCTURES

Received

12 December 2013

Accepted

9 June 2014

Published

11 July 2014

Correspondence and requests for materials should be addressed to S.S. (sshukla@iitb.ac.

\title{
Plasmonic Micro Lens for Extraordinary Transmission of Broadband Light
}

\author{
Sumit Saxena, Raghvendra Pratap Chaudhary, Abhay Singh, Saurabh Awasthi \& Shobha Shukla
}

Nanostructures Engineering and Modeling Laboratory, Metallurgical Engineering \& Materials Science, Indian Institute of Technology Bombay, Mumbai, MH, India 400076.

Extraordinary transmittance and focusing of light in quasi far field region using miniaturized optical devices is a daunting task. A polarization independent, broadband, planar metallic transmissive micro aperture capable of achromatically focusing visible light in quasi far field region is proposed. The calculated enhancement factor of transmission efficiency was about $\sim 2.2$. The total transmission after the aperture is about $60 \%$. This high throughput focusing device will open new avenues for focusing electromagnetic energy in the wide area of sensors and energy concentration. icrolenses are some of the most ubiquitous optical components used in almost all of the light manipulating and focusing devices. Miniaturization of optical lenses is the key limiting factor for advancement of sub-diffraction imaging, ultra high resolution display technology, optical trapping, energy concentration, high density optical information storage and other opto-electronic devices such as sub-micron resolution $^{1,2}$ and molecular detection ${ }^{3}$. Even though refractive micro lenses are available, chromatic and spherical aberrations restrict their applications, their diffractive counterparts on the other hand are extremely difficult to fabricate as they require precise alignment during multiple lithographic processes. A plasmonic microlens based on patches of nanoholes capable of focusing all wavelengths in the visible spectrum to a single spot has recently been proposed ${ }^{4}$ to address the issue of chromatic aberration, however the optical throughput i.e. transmittance of the device was polarization sensitive and restricted to $\sim 5 \%$. These studies have indicated vast potential for application of metal based diffractive optical devices in multicolor stereo imaging, broadband light manipulation and multichannel optical communications.

The phenomenon of extraordinary transmission of light is generally discussed in the context of subwavelength apertures as the enhancement of transmitted light passing through these apertures ${ }^{5}$. One of the very first investigations of extraordinary optical transmission of light through nanometric metallic apertures ${ }^{6,7}$ was reported by Ebbsen et. al. and since then there has been enormous amount of research in the field of metallic nano-optic elements ${ }^{8-14}$. Subsequently investigations revealed an understanding of extraordinary transmission of subwavelength hole arrays ${ }^{15-19}$ and their dependence on hole depth ${ }^{20}$, shape ${ }^{21}$, aperture size and lattice parameters $^{22}$. In year 2008, Verslegers et. al. reported the first experimental demonstration of far-field lensing capability of the plasmonic lenses made of nanoslits in a metallic film ${ }^{23}$. Although it was the first successful demonstration, it could not be commercialized as it required sophisticated focused ion beam milling (FIB) technique which is both expensive and time consuming. A similar attempt was made in the year 2010 in which chirped circular plasmonic lens consisting of corrugated metallic films of silver ${ }^{24}$ and gold ${ }^{25}$ of about $300 \mathrm{~nm}$ and $200 \mathrm{~nm}$ thickness were used. Most of these devices exploit the idea of converting surface plasmons, bound to the metallic surface, to photons as a major contributing phenomenon resulting in a focused beam in the far-field region of the device ${ }^{15,26}$. All of the devices reported to date comprises of nano-slit/hole as a basic building block of planar patch lenses. This limits the total optical throughput, predominantly due to metallic reflection. Ideally, one would like to get as much transmission as possible through the device and focus it to the smallest volume possible to increase the efficiency of the devices using these optical elements.

Here we present a planar metallic microhole lens which uses both, the phenomenon of diffraction as well as surface plasmons to focus the incident light of wavelength ranging from $400 \mathrm{~nm}$ to $700 \mathrm{~nm}$. We primarily exploit the principle of superposition of the incident planar wavefronts with the diffracted non-planar wavefronts ${ }^{27,28}$ which are coupled to surface plasmon waves to generate high energy concentration at the focal spot. This approach gives the freedom to manipulate light with high throughput independent of the lattice geometry or use of expensive lithography techniques. The ability to remove chromatic aberration along with extraordinary transmittance of light will open up new avenues for new type of miniaturized transmissive metal optical devices. 
Full-wave three-dimensional electromagnetic simulation using finite difference time domain (FDTD) method as implemented in Lumerical ${ }^{\circledR}$ was performed. The device response was calculated for wavelengths in the visible range. The design of this metallic planar lens consisting of a double layered optical element comprising of base layer of quartz and top layer of gold with thickenss of $50 \mathrm{~nm}$, was used for simulation purposes. A circular hole of $2.5 \mu \mathrm{m}$ diameter was drilled through the top gold layer. Perfectly matched layer boundary conditions on all the sides of the simulation box were used. The value of permittivity and permeability for the dispersive gold was taken from the reported data by Johnson et. al. ${ }^{29}$. This was illuminated normally by using a planewave with $\mathrm{K}_{0}$ along the $\mathrm{z}$ axis as its propagating vector and the electric field $\mathrm{E}$ pointing along the $\mathrm{x}$-axis, as shown in figure 1(a).

The proposed optical element is circularly symmetric. This enables the formation of focal volume in the form of an ellipsoid, the cross-sectional emission profile of which can be seen in figure 1(b), in the quasi far field region. The focal spot size was calculated by taking the FWHM of the line plot of $|\mathrm{E}|^{2}$ across the aperture cross-section and found to depend on the wavelength as shown in figure 1(c). Our FDTD simulations show that the focal spot size, depth of focus and focal length were found to depend on the aperture size and is in agreement with the data reported for nanohole patches $^{4}$ (supplementary Figure S8 in the Reference 4). The focal length of the microhole lens was calculated from the Rayleigh Sommerfield integral by requiring that

$$
\frac{d I}{d z}=-2 A_{0} \frac{\pi \rho^{2}}{\lambda z^{2}} \operatorname{Sin}\left(\frac{\pi \rho^{2}}{\lambda z}\right)=0
$$

Here $\rho$ represents the radius of the circular aperture while $\lambda$ represents the operating wavelength. The calculated focal lengths $(\mathrm{z})$ using the above expression at operating wavelength in the range $400 \mathrm{~nm}-$ $700 \mathrm{~nm}$ have been plotted in figure 1(c). These values were in good agreement to the values obtained using FDTD techniques. It is inter- esting to note that all wavelengths in the visible range were focused within $\sim 1.5 \mu \mathrm{m}$ is significantly better than those for nanohole patches. The depth of focus also reduces to about $1 / 8^{\text {th }}$ of that of the nanohole patch ${ }^{4}$. Since the shift of the focal length is considerably smaller than the depth of the focus, figure 1(c) suggests that these microlenses will act as better broadband focusing elements as compared to the nanohole arrays. In general, the far field focusing does not contain the evanescent field information, the focal spot size is subject to classical diffraction limit ${ }^{30}$. The maximum size of the focal spot was $\sim 720 \mathrm{~nm}$ around wavelength of $495 \mathrm{~nm}$ (b). This is very close to the classical theoretical minimum diffraction limited spot size $\sim 760 \mathrm{~nm}$ estimated by the relation $d=\frac{1.22 \lambda z}{2 \rho}$. The minimum spot size was observed to be $\sim 645 \mathrm{~nm}$ at the two extremes of the scanned wavelength. The focal spot width obtained from circular aperture is much less than that of nanohole array $(1.83 \mathrm{um})$ and other 2D array of spatially varying apertures ${ }^{31}$.

The interactions of the electromagnetic radiation at the metaldielectric interface excite surface plasmons at both, the gold - quartz Interface as well as the gold - air interfaces and get coupled through the hole. These surface plasmons then reradiate into photons resulting in very high enhancement factor for transmission efficiency, which is estimated to be $\sim 2.2$ for $2.5 \mu \mathrm{m}$ gold aperture. The comparison of the intensity at the focal spots in figure 2(a)(1) and 2(b)(1) suggests that this is strongly dependent on the incident wavelength and is maximum when the incident wavelength matches the surface plasmon resonance of the circular aperture. The distribution of $|\mathrm{Ez}|^{2}$ in figures $2(\mathrm{a})(4)$ and 2(b)(4) appears to be tied and localized on both sides of the aperture implying the occurrence of charge densities. These fields are symmetrical and found to be more intense for longer wavelength in gold. The presence of surface plasmons in these metallic micro apertures is also observed in figure $3(\mathrm{a})$. The distribution of $|\mathrm{Ex}|^{2}$ in figures 2(a)(1) and 2(b)(1) gives a clear indication of the long range coupled surface plasmon mode (waveguide mode).

Further analysis of the field distribution shows the formation of surface plasmon wave with a wave length of $\sim 250 \mathrm{~nm}$ at both the (a)

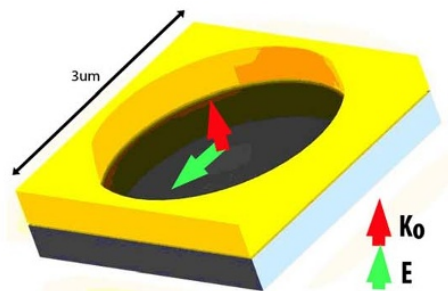

(c) 10

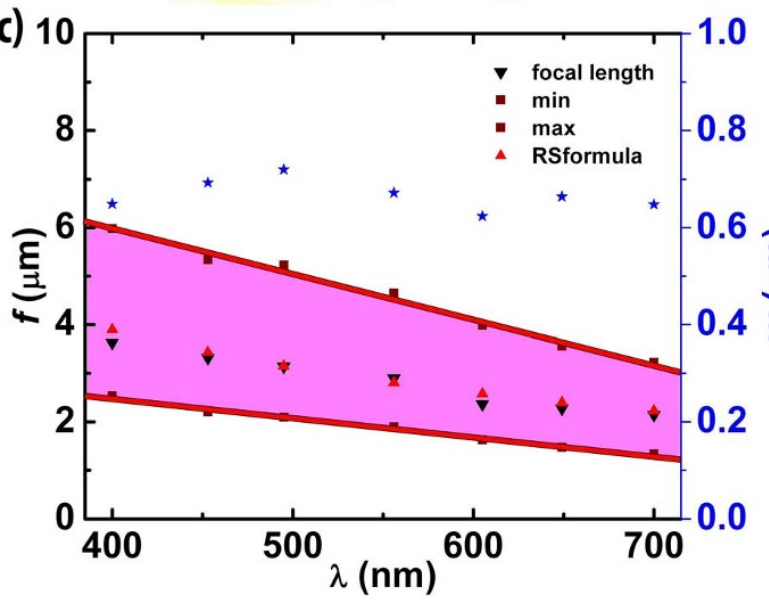

(b)

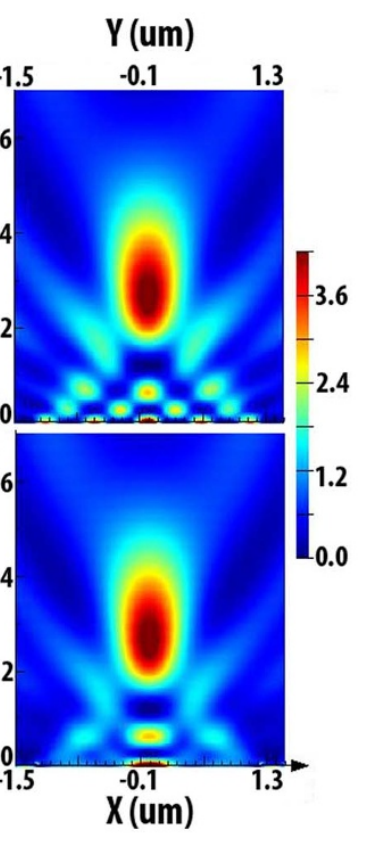

Figure $1 \mid$ (a) Schematic of broadband, planar metallic lens. The top layer is a patterned gold layer of thickness $50 \mathrm{~nm}$ and the base layer is of quartz. The propagation vector $\mathrm{K}_{0}$ is normally incident to the lens, while the direction of $\mathrm{E}$ field is normal to $\mathrm{K}_{0}$. (b) Emission profile at $\lambda=580 \mathrm{~nm}$ along the $\mathrm{xz}$ and yz planes. Both graphs are on same color scale. (c) Calculated focal length $(f)$ and focal spot size (w) indicated by "*” using FDTD simulations. The change in focal length was smaller than that of depth of the focus. The depth of focus has been shaded as a guide for eye. 

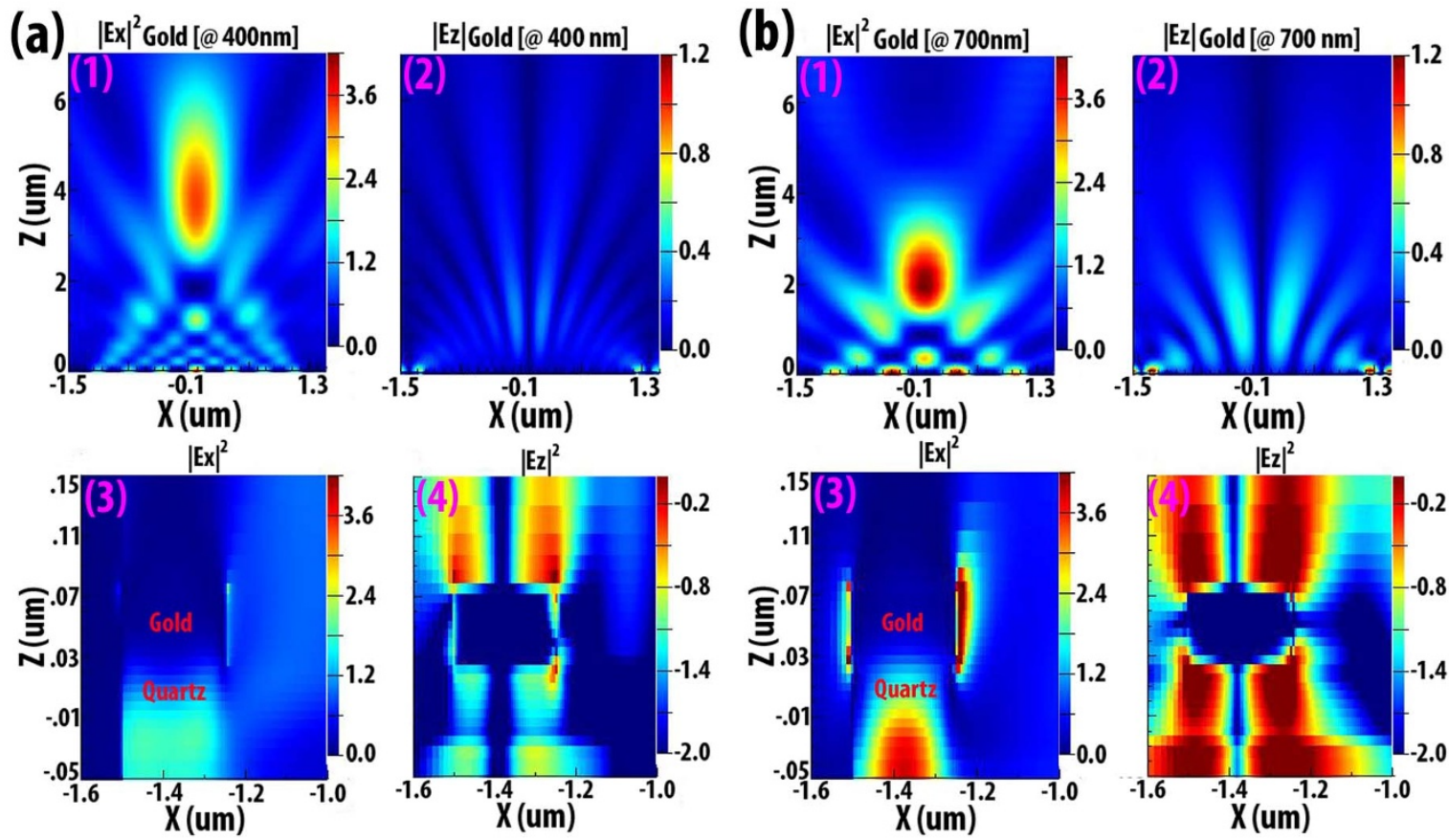

Figure 2 Cross-sectional time averaged emission profile of circular aperture metallic lens for gold at 400 and $700 \mathrm{~nm}$. Graphs (1), (2) and (3), (4) have been plotted on same length $Y$ scale respectively. $\mathrm{a}(1)$ and $\mathrm{b}(1)$ represent the distribution of $\left|\mathrm{E}_{\mathrm{x}}\right|^{2}$ at $400 \mathrm{~nm}$ and $700 \mathrm{~nm}$. $\mathrm{a}(2)$ and $\mathrm{b}(2)$ represent the distribution of $\left|\mathrm{E}_{\mathrm{z}}\right|$ at $400 \mathrm{~nm}$ and $700 \mathrm{~nm} . \mathrm{a}(3)$ and $\mathrm{a}(4)$ represent the zoomed images for $\left|\mathrm{E}_{\mathrm{x}}\right|^{2}$ and $\left|\mathrm{E}_{\mathrm{z}}\right|^{2}$ at $400 \mathrm{~nm}$ respectively, while $\mathrm{b}(3)$ and $\mathrm{b}(4)$ represent the same at $700 \mathrm{~nm}$. The spatial positions of gold and quartz substrate are same in (3) and (4) for both (a) and (b). The color scale for figure (a)(4) and (b)(4) are on logarithmic scales.

quartz - gold and gold - air interfaces, and was found to be constant for incident wave with wavelength $400 \mathrm{~nm}-700 \mathrm{~nm}$. These observations are not in accordance with the observations in nano hole arrays with large lattice constants in which wavelength of surface plasmon waves were reported to be $\lambda_{\text {spp }} / 2$ at the gold - air interface ${ }^{32,33}$. These can be understood in analogy with the formation of waves on a string of fixed length tied at both ends. The linear dimensions of the metal dielectric interface is almost of the same dimensions as $\lambda_{\text {spp }} / 2$. This forces the surface plasmon waves to limit its wavelength to the dimensions of the metal-dielectric interface. In order to test this hypothesis, the lattice constant of the cell was increased significantly and the observed results were in accordance with that of their nanohole counterparts ${ }^{32}$. The transmission curve in figure 3(b) suggests that the proposed microholes can be used for enhanced transmission over the entire visible spectrum. The maximum transmission of $\sim 60 \%$ is observed at $580 \mathrm{~nm}$ as seen in the inset in figure 3(b).
In order to further investigate the contribution of surface plasmons to the extraordinary transmission, the time averaged intensity of the surface plasmon waves was estimated using the methodology proposed by Mehfuz et. al. ${ }^{34}$. It is observed that the absolute contribution of the intensity due to surface plasmons remains almost the same in both the nanohole and microhole apertures. The Intensity of the radiant mode $\left(I_{r}\right)$ however increases significantly in microhole apertures as compared to collective contribution of nano apertures in micropatches, because clear micro apertures allow more light to pass through without any reflection losses. The coupling efficiency of the surface plasmons wave is inversely proportional to $I_{r}$, and since the nanohole apertures, by design, restrict the transmission of light, their coupling efficiencies become larger. The coupling efficiency calculated by incorporating the radiant mode is understood to provide a measure of contribution due to the phenomenon associated predominantly with the surface plasmons in nano aperture patches.
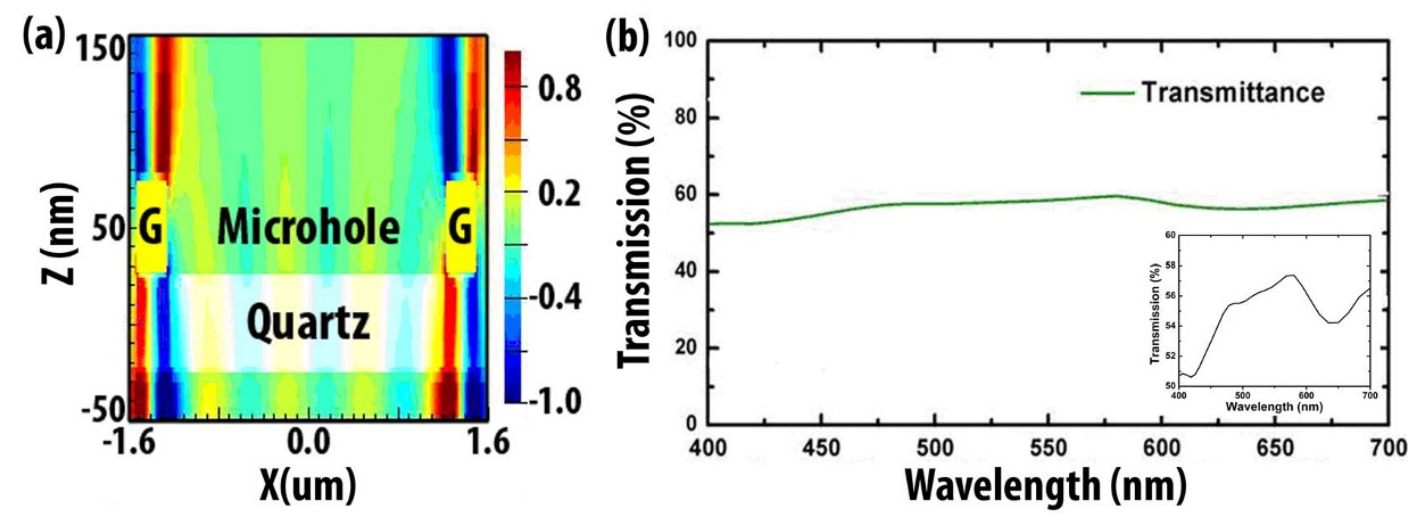

Figure $3 \mid$ (a) Cross-sectional time averaged E profile of circular aperture for gold at $700 \mathrm{~nm}$. "G" represents gold. (b) Calculated total transmittance for the microhole aperture in the frequency range $400 \mathrm{~nm}-700 \mathrm{~nm}$. The inset shows the variation of transmittance with frequency. 


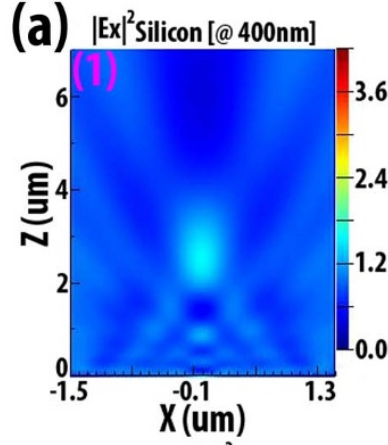

$|\mathrm{Ex}|^{2}$

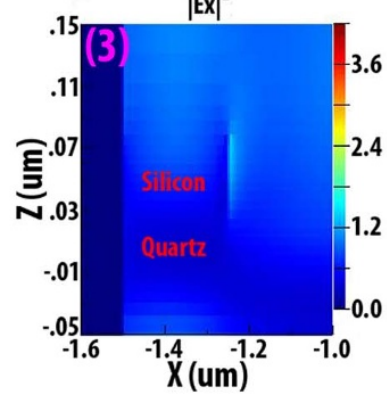

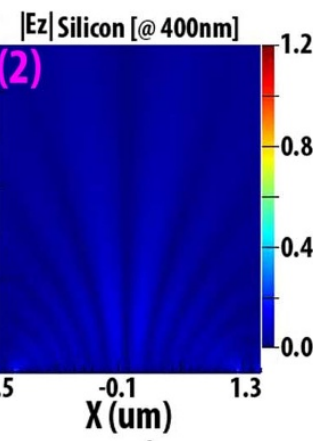

$|\mathrm{Ez}|^{2}$

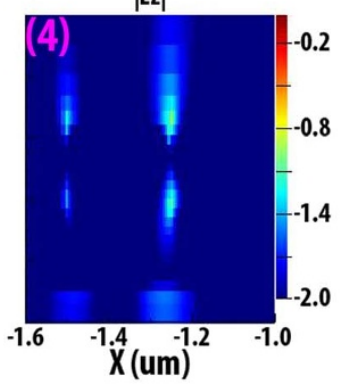

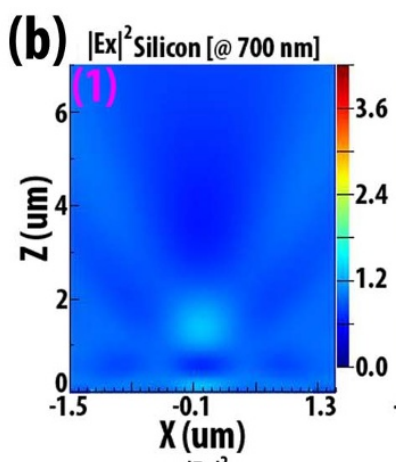

$|\mathrm{Ex}|^{2}$

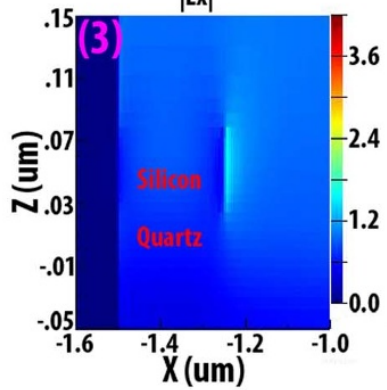

|Ez|Silicon [@ $700 \mathrm{~nm}]$

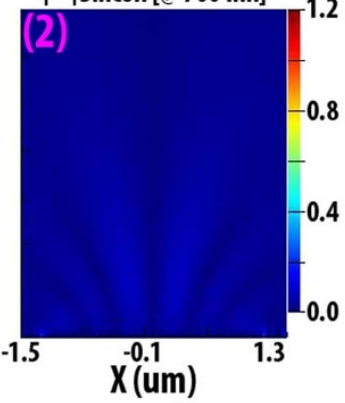

$|E z|^{2}$

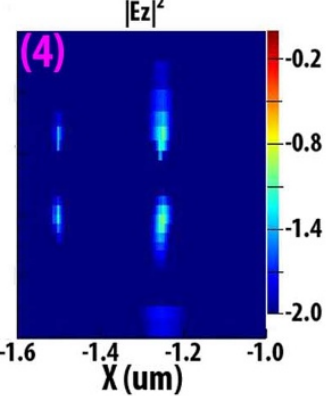

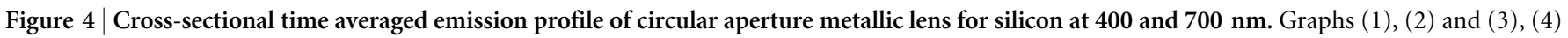
have been plotted on same length $\mathrm{Y}$ scale respectively. (a)(1) and (b)(1) represent the distribution of $\left|\mathrm{E}_{\mathrm{X}}\right|^{2}$ at $400 \mathrm{~nm}$ and $700 \mathrm{~nm}$. (a)(2) and (b)(2)

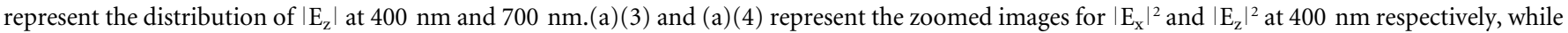

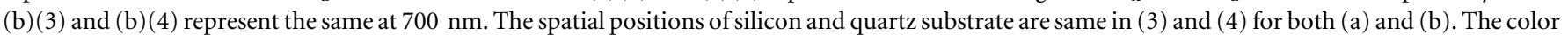
scale for figure (a)(4) and (b)(4) are on logarithmic scales.

Although by definition of coupling efficiency, the microholes does not do as good as nanoholes, the contribution due to the transmission and diffraction improves the performance of the microholes over their nano cousins. A further increase in the aperture size will make plasmonic effects as minor perturbative effects.

The phenomenon of surface plasmons is understood to be supported on metal dielectric interface ${ }^{33}$, calculations were repeated for other metals (Supplementary figure S1-S2) along with well known non-plasmonic material such as Silicon, to establish the role played by surface plasmons in the extraordinary transmission efficiency of microhole metal lenses. Comparison of the intensities of the $|\mathrm{Ex}|^{2}$ and $|\mathrm{Ez}|$ emission profile for silicon in figure 4 with that of gold in figure 2 clearly shows the contribution of the photons emitted from surface plasmons in enhancement of intensities of electromagnetic radiations in the case of gold microholes.

\section{Conclusion}

In a nutshell, we have presented an extraordinarily transmissive, polarization independent, broadband planar metallic lens. The proposed optical element is simple and can be fabricated using standard lithographic techniques and shows excellent achromatism in the visible region. The extraordinarily high transmission in these microhole lenses is understood to be predominantly due to reduced metal surface area, while the surface plasmons along with diffraction is understood to be responsible for its high enhancement factor of transmission efficiency. Further modifications in design are expected to achieve super resolution using these plasmonic lenses thereby revolutionizing many areas of super resolution microscopy, single molecule spectroscopy to name a few.

1. Huang, F. M., Zheludev, N., Chen, Y. \& Javier Garcia de Abajo, F. Focusing of light by a nanohole array. Appl. Phys. Lett. 90, 091119 (2007).

2. Rogers, E. T. F. et al. A super-oscillatory lens optical microscope for subwavelength imaging. Nature Mater. 11, 432 (2012).

3. De Angelis, F. et al. A hybrid plasmonic-photonic nanodevice for label-free detection of a few molecules. Nano Lett. 8, 2321 (2008).
4. Gao, H. et al. Broadband plasmonic microlenses based on patches of nanoholes. Nano Lett. 10, 4111 (2010)

5. Coe, J. V., Heer, J. M., Teeters-Kennedy, S., Tian, H. \& Rodriguez, K. R. Extraordinary transmission of metal films with arrays of subwavelength holes. Annu. Rev. Phys. Chem. 59, 179 (2008).

6. Ebbesen, T. W., Lezec, H. J., Ghaemi, H. F., Thio, T. \& Wolff, P. A. Extraordinary optical transmission through sub-wavelength hole arrays. Nature 391, 667 (1998).

7. Ghaemi, H. F., Thio, T., Grupp, D. E., Ebbesen, T. W. \& Lezec, H. J. Surface plasmons enhance optical transmission through subwavelength holes. Phys. Rev. B 58, 6779 (1998).

8. Sun, Z. \& Kim, H. K. Refractive transmission of light and beam shapingwith metallic nano-optic lenses. Appl. Phys. Lett. 85, 642 (2004)

9. Shi, H. et al. Beam manipulating by metallic nano-slits with variant widths. Opt. Express 13, 6815 (2005).

10. Min, C., Wang, P., Jiao, X., Deng, Y. \& Ming, H. Beam manipulating by metallic nano-optic lens containing nonlinear media. Opt. Express 15, 9541 (2007).

11. Huang, F. M., Kao, T. S., Fedotov, V. A., Chen, Y. \& Zheludev, N. I. Nanohole array as a lens. Nano Lett. 8, 2469 (2008).

12. Przybilla, F., Genet, C. \& Ebbesen, T. W. Enhanced transmission through penrose subwavelength hole arrays. Appl. Phys. Lett. 89, 121115 (2006).

13. Ishii, S., Shalaev, V. M. \& Kildishev, A. V. Holey-Metal Lenses: Sieving single modes with proper phases. Nano Lett. 13, 159 (2013).

14. Torres, V. et al. Terahertz epsilon-near-zero graded-index lens. Opt. Express 21, 9156 (2013).

15. Martin-Moreno, L. et al. Theory of extraordinary optical transmission through subwavelength hole arrays. Phys. Rev. Lett. 86, 1114 (2001).

16. Sarrazin, M. \& Vigneron, J.-P. Optical properties of tungsten thin films perforated with a bidimensional array of subwavelength holes. Phys. Rev. E 68, 016603 (2003).

17. Schroter, U. \& Heitmann, D. Surface-plasmon-enhanced transmission through metallic gratings. Phys. Rev. B 58, 15419 (1998).

18. Sarrazin, M. \& Vigneron, J.-P. Light transmission assisted by Brewster-Zennek modes in chromium films carrying a subwavelength hole array. Phys. Rev. B 71, 075404 (2005).

19. Liu, H. \& Lalanne, P. Microscopic theory of the extraordinary optical transmission. Nature 452, 728 (2008).

20. Degiron, A., Lezec, H. J., Barnes, W. L. \& Ebbesen, T. W. Effects of hole depth on enhanced light transmission through subwavelength hole arrays. Appl. Phys. Lett. 81, 4327 (2002).

21. Chen, C.-Y., Tsai, M.-W., Chuang, T.-H. \& Li, S.-C. Extraordinary transmission through a silver film perforated with cross shaped hole arrays in a square lattice. Appl. Phys. Lett. 91, 063108 (2007).

22. van der Molen, K. L. et al. Role of shape and localized resonances in extraordinary transmission through periodic arrays of subwavelength holes: Experiment and theory. Phys. Rev. B 72, 045421 (2005). 
23. Verslegers, L. et al. Planar lenses based on nanoscale slit arrays in a metallic film. Nano Lett. 9, 235 (2008).

24. Fu, Y., Zhou, W., Lim, L. E. N., Du, C. L. \& Luo, X. G. Plasmonic microzone plate: Superfocusing at visible regime. Appl. Phys. Lett. 91, 061124 (2007).

25. Fu, Y., Liu, Y., Zhou, X., Xu, Z. \& Fang, F. Experimental investigation of superfocusing of plasmonic lens with chirped circular nanoslits. Opt. Express 18, 3438 (2010).

26. Lezec, H. J. et al. Beaming light from a subwavelength aperture. Science 297, 820 (2002).

27. Xi, Y., Jung, Y. S. \& Kim, H. K. Interaction of light with a metal wedge: the role of diffraction in shaping energy flow. Opt. Express 18, 2588 (2010).

28. Barnes, W. L., Dereux, A. \& Ebbesen, T. W. Surface plasmon subwavelength optics. Nature 424, 824 (2003).

29. Johnson, P. B. \& Christy, R. W. Optical constants of the noble metals. Phys. Rev. B 6, 4370 (1972).

30. Liu, Z. et al. Far-field optical superlens. Nano Lett. 7, 403 (2007)

31. Lin, L., Goh, X. M., McGuinness, L. P. \& Roberts, A. Plasmonic Lenses Formed by Two-dimensional nanometric cross-shaped aperture arrays for fresnel-region focusing. Nano Lett. 10, 1936 (2010).

32. Yin, L. et al. Surface plasmons at single nanoholes in Au films. Appl. Phys. Lett. 85, 467 (2004).

33. Gao, H., Henzie, J. \& Odom, T. W. Direct evidence for surface plasmon-mediated enhanced light transmission through metallic nanohole arrays. Nano Lett. 6, 2104 (2006)

34. Mehfuz, R., Maqsood, M. W. \& Chau, K. J. Enhancing the efficiency of slitcoupling to surface-plasmon-polaritons via dispersion engineering. Opt. Express 18, 18206 (2010)

\section{Acknowledgments}

This work was supported by the Department of Science and Technology, Solar Energy Research Initiative (SERI), Government of India grant via sanction order no. DST/TM/ SERI/2k10/12/(G) and the Industrial Research and Consultancy Services, Indian Institute of Technology Bombay, grant no. (11IRCCSG025).

\section{Author contributions}

Sh.S., A.S. and Su.S. conceived the idea and concept. R.C., S.A. and A.S. performed the simulations and generated the data. Su.S., S.A. and R.C. analyzed the data. Su.S. and Sh.S. wrote the manuscript.

\section{Additional information}

Supplementary information accompanies this paper at http://www.nature.com/ scientificreports

Competing financial interests: The authors declare no competing financial interests.

How to cite this article: Saxena, S., Chaudhary, R.P., Singh, A., Awasthi, S. \& Shukla, S. Plasmonic Micro Lens for Extraordinary Transmission of Broadband Light. Sci. Rep. 4, 5586; DOI:10.1038/srep05586 (2014).

This work is licensed under a Creative Commons Attribution-NonCommercialNoDerivs 4.0 International License. The images or other third party material in this article are included in the article's Creative Commons license, unless indicated otherwise in the credit line; if the material is not included under the Creative Commons license, users will need to obtain permission from the license holder in order to reproduce the material. To view a copy of this license, visit http:// creativecommons.org/licenses/by-nc-nd/4.0/ 